\title{
Exploring the Potential of a Questionnaire in Predicting Results of Nerve Conduction Studies in Patients with Suspected Carpal Tunnel Syndrome: Exploring a Clinician and Patient Complete Version
}

\author{
Carl Edwards \\ Torbay and South Devon NHS Foundation Trust, Torquay, UK \\ Email: carl.edwards@nhs.net
}

How to cite this paper: Edwards, C. (2020) Exploring the Potential of a Questionnaire in Predicting Results of Nerve Conduction Studies in Patients with Suspected Carpal Tunnel Syndrome: Exploring a Clinician and Patient Complete Version. Open Journal of Therapy and Rehabilitation, 8, 110-118. https://doi.org/10.4236/ojtr.2020.83010

Received: February 26, 2020

Accepted: August 9, 2020

Published: August 12, 2020

Copyright $\odot 2020$ by author(s) and Scientific Research Publishing Inc. This work is licensed under the Creative Commons Attribution International License (CC BY 4.0).

http://creativecommons.org/licenses/by/4.0/

\begin{abstract}
This evaluation of the Carpal Tunnel Questionnaire (CTQ) was designed to establish the validity and flexibility of its potential use both within a primary and secondary care setting. The questionnaire was originally designed to predict the positive and negative outcome of Nerve Conduction Studies (NCS) in those patients with suspected Carpal Tunnel Syndrome (CTS). Prior to being seen by the hand specialist patients who had been referred with suspected CTS where asked to complete the CTQ. These questionnaires were coded and filed by the clinic nurse. The hand specialist then completed the questionnaire with the patient preceding NCS. Questionnaires were scored subsequent to the appointment. Results for the hand specialist completed questionnaire showed an $80 \%$ specificity and $92 \%$ sensitivity regarding the ability of the CTQ to predict a positive NCS using a pre-determine cut-off score. The patient completed questionnaire showed a $70.67 \%$ specificity and $72 \%$ sensitivity. Using receiver operating characteristics a threshold score could be determined to achieve $100 \%$ sensitivity/specificity for both questionnaires. This questionnaire provides a useful addition in the assessment of patients with suspected carpal tunnel syndrome and could be used in a range of clinical settings although the scoring cut-off may need to be adapted depending on whether the questionnaire was completed by the clinician or patient. Using the questionnaire in a clinical setting would reduce the requirement for NCS by $60 \%$, this would offer significant time and cost savings.
\end{abstract}

\section{Keywords}

Carpal Tunnel Syndrome, Questionnaire, Nerve Conduction Studies, Primary/Secondary Care 


\section{Introduction}

Carpal Tunnel Syndrome (CTS) is the most common entrapment neuropathy presenting to the Orthopaedic outpatient department affecting between $5 \%-16 \%$ of the population [1]. Assessment of CTS depends on detailed history taking, clinical examination and nerve conduction studies (NCS). NCS have traditionally been viewed as the most effective method for the assessment of CTS; however, they carry a cost and often delay treatment due to additional waiting times. In any case, NCS however have limitations (such as false positive and false negative results) and so need to be interpreted alongside other clinical data; there is no single "gold standard" test in the diagnosis of CTS.

A questionnaire developed by Kamath and Stothard (2003) based on original work by Levine et al. (1993) has been shown to demonstrate a high sensitivity when compared to positive results from NCS [2] [3] [4] and outcome from carpal tunnel decompression surgery [5]. The 9-item questionnaire is scored using an algorithm with items being weighted differently. This questionnaire however has only been assessed when being completed by a hand specialist and not used as a patient-completed questionnaire, thus limiting its potential application within primary care settings as a component of screening for CTS.

The aim of this study is to explore the positive and negative predictive validity of a clinician and patient-completed version of the CTQ against the outcome of subsequent NCS. A secondary aim is to explore the scoring algorithm through logistic regression.

\section{Research Methods}

\subsection{Participants}

100 consecutive patients attending an orthopaedic hand clinic with suspected Carpal Tunnel Syndrome (CTS) who met the inclusion criteria were selected for the study.

The cohort of participants for the current study was acquired through direct referral from either primary care (usually their own General Practitioner) or through secondary care via other specialist clinics. These patients were referred with the direct question as to whether they did, or did not have CTS. Power was determined through reviewing previous research including the original study carried out by Levine et al. (1993) exploring the use of a questionnaire in the assessment of CTS $(\mathrm{n}=67)$. The questionnaire developed and evaluated by Kamath and Stothard (2003) included 107 consecutive patients referred into a hand clinic with suspected CTS, of whom 74 met the inclusion criteria and 16 were lost to follow up, giving a total sample size in that study of 58 .

\subsection{Inclusion Criteria}

- Those able to offer informed consent

- Patients having been referred with suspected Carpal Tunnel Syndrome

- Patient aged over 18 year (no upper age limit)

- Those patients who are suitable for NCS 


\subsection{Exclusion Criteria}

- Peripheral Neuropathy including those diagnosed with Diabetes Mellitus

- Pregnancy

- Patients who have undergone renal transplant

- Previous Carpal Tunnel Decompression on the same side

- Those unable to give informed consent

- Those under the age of 18 years

Diagnosis of CTS in patients with diabetic neuropathy is difficult as the two conditions may affect the median nerve in a similar way. Renal transplant patients (often requiring more involved surgery including the removal of amyloid tissue) and pregnant patients were also excluded. Carpal tunnel syndrome can recur but it is rare and this may well complicate matters; in any case all patients returning to an orthopaedic clinic with recurrence of symptoms would normally require repeat NCS.

Prior to the commencement of the clinic, notes were reviewed and those meeting the inclusion criteria were selected. Those patients deemed appropriate for the study were provided with a patient information leaflet and questionnaire. It was made specifically clear at this point that their decision to consent to the study would have no bearing on the outcome of their clinic appointment.

\subsection{Study Site}

All patients were seen within the orthopaedic outpatient department at Torbay and South Devon NHS Foundation Trust (2014-15).

\subsection{Procedure}

Patients meeting the inclusion criteria were provided with the patient information leaflet and questionnaire upon their attendance. Sufficient time was offered in order for the patients to decide whether or not they wished to participate in the study.

Patient-completed questionnaires were posted into a sealed box and were not seen by the hand specialist. Participants then underwent a standard clinical examination and completion of the CTQ this time administered by the hand specialist. NCS were carried out on all patients. Questionnaire responses were not analysed until a later date and had no influence over the clinical management of the patient.

Criteria for the interpretation of NCS were based on the Kamath and Stothard (2003) study. Criteria for normal values were matched, with terminal latency to abductor pollicis brevis less than $4.0 \mathrm{~ms}$ and a sensory conduction from digit 2 to wrist greater than $47 \mathrm{~m} / \mathrm{s}$. Further routine tests included transpalmar recording to digit 3 with a $20 \%$ reduction in conduction velocity for the median nerve across the carpal tunnel compared to the palm to finger recording considered significant.

\subsection{Materials}

The CTQ consists of nine questions related to the common symptoms reported by patients suffering with CTS. The questions are differentially weighted giving a 
possible scoring range of between -2 and +11 . Both the questionnaire used within the study and scoring system are included in Appendix.

\subsection{Data Analysis}

Total clinician completed and patient-completed CTQ scores for each participant were computed using the original weighting algorithm described by Kamath and Stothard (2003). Using the original threshold of CTQ weighted score $\geq 5$, specificity and sensitivity together with positive and negative predictive values in relation to obtained positive NCS results were derived. Based on the results of Edwards and Frampton (2014), the effect of raising the threshold to $\geq 6$ were analysed in the same way. Receiver operator characteristic (ROC) analysis was used to derive a single optimum cut off questionnaire score, in order to establish the positive and negative predictive validity of the patient-completed questionnaire in relation to the outcome of subsequent NCS. Logistic regression was utilised to explore the validity of the scoring algorithm. Raw scores on the CTQ for each participant were then entered into a binary logistic model predicting NCS outcome (positive or negative). Beta coefficients were extracted for each item in the questionnaire and applied as a weighting.

\section{Results}

\subsection{Clinician-Completed Version}

Clinician-completed CTQ weighted total scores were calculated for 100 patients, of whom 75 (75\%) subsequently tested positive for CTS on NCS, 25 (25\%) tested negative. Inspection of the results (Figure 1) suggests that all those patients scoring 7 or over $(n=31)$ on the questionnaire had positive NCS for CTS. Of those who scored $\geq 6$ ( $n=54)$ only one patient subsequently obtained negative NCS.

Using the original Kamath and Stothard (2003) cut-off score of $\geq 5,60 \%$ ( $\mathrm{n}=$ $60)$ scored $\geq 5$ with seven of those (11.7\%) with negative NCS and $53(88.3 \%)$ had positive NCS. Table 1 records the sensitivity, specificity and positive/negative predictive validity of these thresholds.

ROC analysis (Figure 2) demonstrated significantly positive results for the questionnaire when considering its use as a screening tool for NCS.

\subsection{Patient-Completed Version}

Patient-completed CTQ weighted total scores were calculated for the same 100 patients, of whom 75 (75\%) subsequently tested positive for CTS on NCS, 25 (25\%) tested negative. Inspection of the results (Figure 3) suggests that all but one of those patients scoring 7 or over $(n=26)$ on the questionnaire had positive tests for CTS. Of those who scored $\geq 6(n=43)$ only two patients subsequently obtained negative NCS.

Using the original Kamath and Stothard (2003) cut-off score of $\geq 5,60 \%$ ( $\mathrm{n}=$ $60)$ scored $\geq 5$ with seven of those (11.7\%) negative NCS and 53 (88.3\%) had positive NCS. Table 2 records the sensitivity, specificity and positive/negative predictive validity of these thresholds. 
ROC analysis demonstrated significantly positive results for the questionnaire when considering its use as a screening tool for NCS. Figure 4 shows the ROC curve. The area under the curve was calculated depicting how well the questionnaire

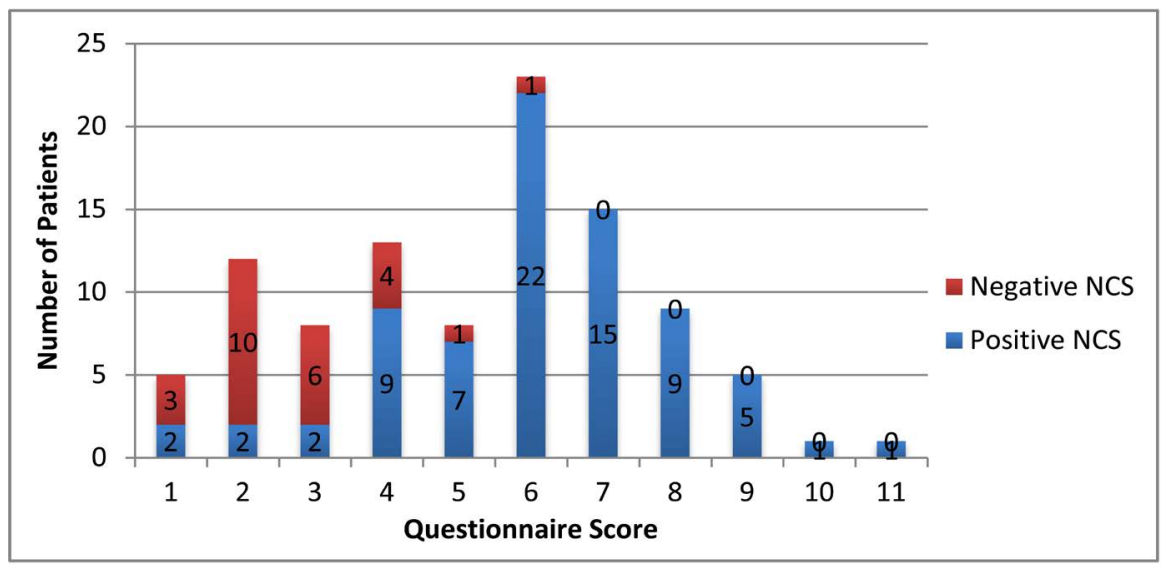

Figure 1. The distribution of weighted total clinician-completed Carpal Tunnel Questionnaire scores obtained.

Table 1. Sensitivity/specificity and positive/negative predictive values of the CTQ relative to results of NCS using $\geq 5, \geq 6$ and $\geq 7$ as cut-off scores.

\begin{tabular}{cccccc}
\hline $\begin{array}{c}\text { Cut-off } \\
\text { score }\end{array}$ & Specificity & Sensitivity & $\begin{array}{c}\text { Positive } \\
\text { Predictive Value }\end{array}$ & $\begin{array}{c}\text { Negative } \\
\text { Predictive Value }\end{array}$ & $\begin{array}{c}\text { False Positive } \\
\text { Rate }\end{array}$ \\
\hline 5 or over & $80 \%$ & $92 \%$ & $96.77 \%$ & $60.53 \%$ & $2 \%(\mathrm{n}=2)$ \\
6 or over & $70.67 \%$ & $96 \%$ & $98.15 \%$ & $52.17 \%$ & $1 \%(\mathrm{n}=1)$ \\
7 or over & $41.33 \%$ & $100 \%$ & $100 \%$ & $36.23 \%$ & $0 \%$ \\
\hline
\end{tabular}

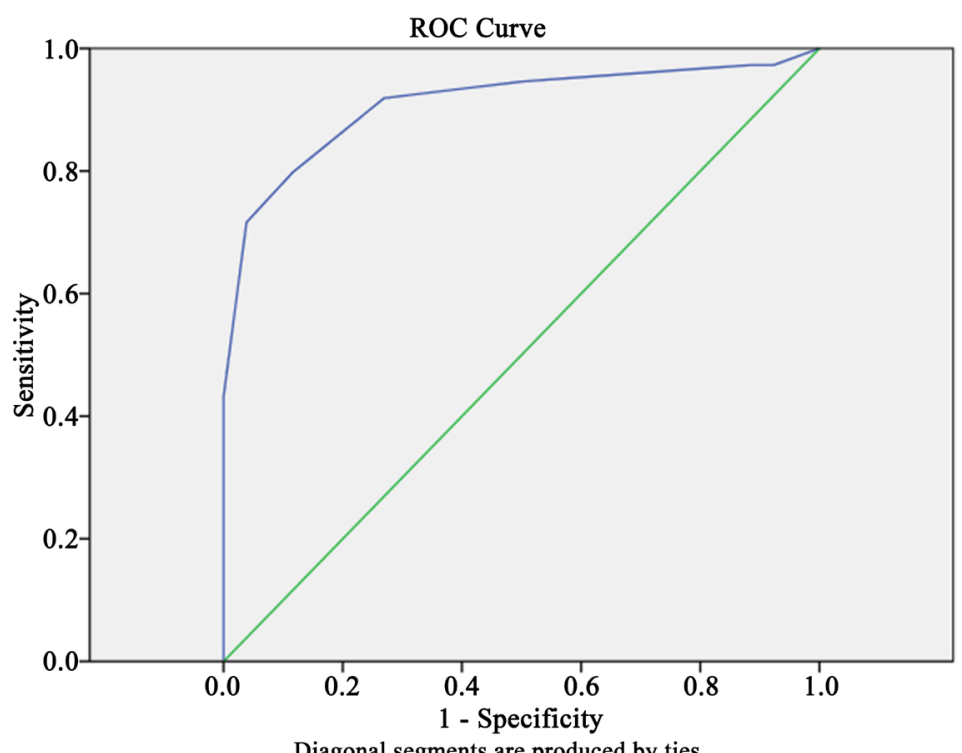

Figure 2. Receiver Operating Characteristic (ROC) analysis of alternative cut-off scores on hand clinician-completed Carpal Tunnel Questionnaire for predicting outcome of nerve conduction studies. 


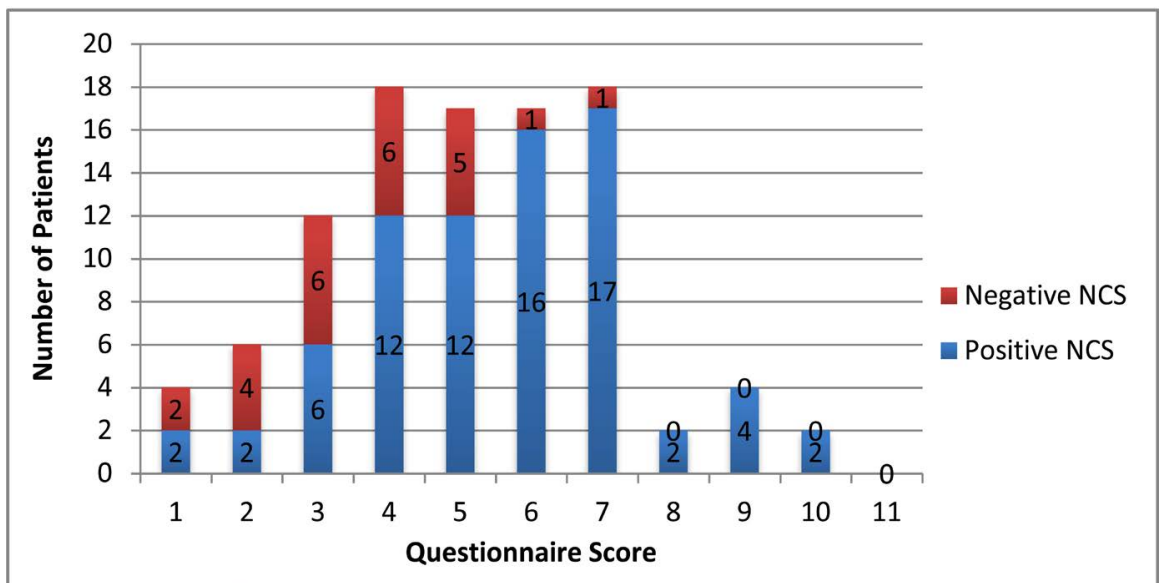

Figure 3. Distribution of weighted total patient-completed carpal tunnel questionnaire scores obtained.

Table 2. Sensitivity/specificity and positive/negative predictive values of the patientcomplete CTQ relative to results of NCS using $\geq 5, \geq 6$ and $\geq 7$ as cut-off scores.

\begin{tabular}{cccccc}
\hline $\begin{array}{c}\text { Cut-off } \\
\text { score }\end{array}$ & Specificity & Sensitivity & $\begin{array}{c}\text { Positive Predictive } \\
\text { Value }\end{array}$ & $\begin{array}{c}\text { Negative } \\
\text { Predictive Value }\end{array}$ & $\begin{array}{c}\text { False Positive } \\
\text { Results }\end{array}$ \\
\hline 5 or above & $70.67 \%$ & $72 \%$ & $88.33 \%$ & $45.00 \%$ & $7 \%(\mathrm{n}=7)$ \\
6 or above & $54.67 \%$ & $92 \%$ & $95.35 \%$ & $40.35 \%$ & $2 \%(\mathrm{n}=2)$ \\
7 or above & $33.33 \%$ & $96 \%$ & $96.15 \%$ & $32.43 \%$ & $1 \%(\mathrm{n}=1)$ \\
\hline
\end{tabular}

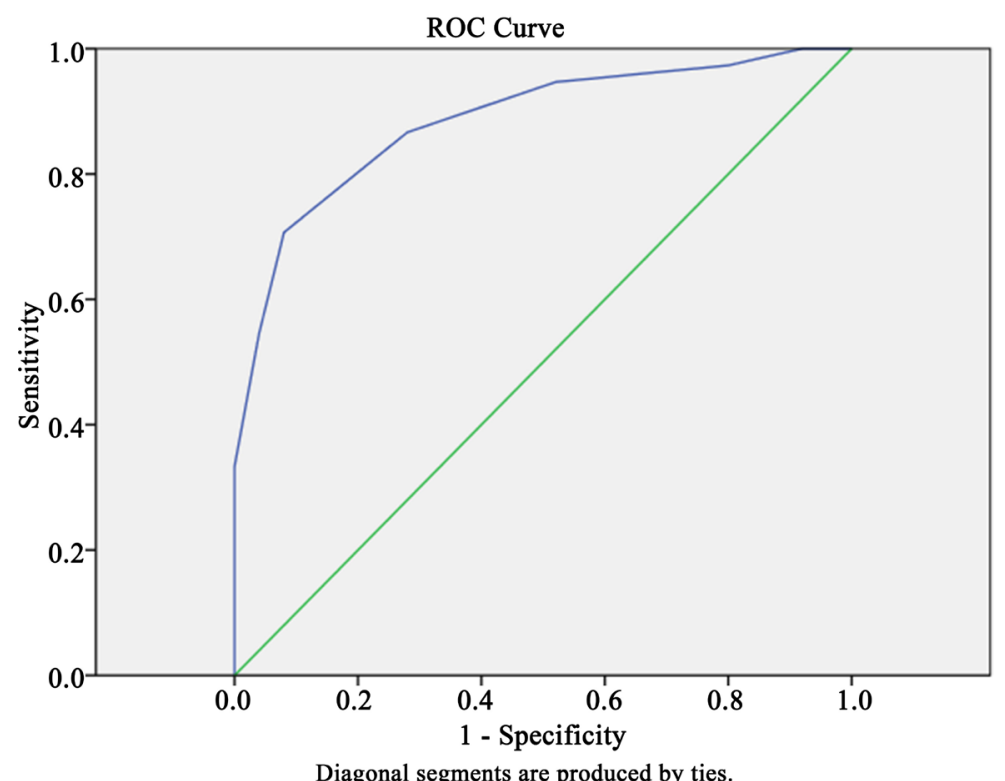

Figure 4. ROC analysis of alternative cut-off scores patient-completed version of the CTQ.

can distinguish between the two diagnostic groups (positive NCS/negative NCS) based on a range of possible threshold scores. The total area under the curve $(0.885,95 \%$ confidence interval 0.816 to 0.953$)$ indicates that the predictive validity of the questionnaire is statistically significant $(\mathrm{p}<0.001)$. 


\subsection{Scoring Algorithm}

The original scoring is simple; easy to be carried out in a clinic setting and produces a high level of specificity. The revised scoring algorithm obtaining through using a regression model did not improve the function of the CTQ.

\section{Discussion}

In a study of the clinician-complete and patient-completed versions of the Carpal Tunnel Questionnaire (CTQ) in 100 participants, sensitivity, specificity and positive/negative predictive validity coefficients were derived for three cut-off thresholds for weighted totals, based on Kamath and Stothard (2003). ROC analyses confirmed that all reviewed potential cut-off thresholds (5 - 7) were significantly better than chance at predicting the outcome of nerve conduction studies (NCS). Inspection of the ROC curve indicates the optimum balance between sensitivity and specificity, such that false negatives (those who are below threshold for the screening questionnaire but subsequently test positive on NCS) are equally important as false positives (those above the threshold for the screening questionnaire who subsequently test negative on NCS).

In clinical practice, the relative balance between false positives and false negatives depends on the consequences of subsequent investigations and ultimately surgical intervention. If a patient were to score below the questionnaire threshold they will go on to have confirmatory NCS and therefore the false negative rate is not of a clinical concern. The sensitivity/true positive rate is of significance; when relying on the questionnaire as a potential screening tool it is crucial that those scoring above the threshold would definitely have subsequent positive NCS (if questionnaire results are being used to omit these studies). The results of the current study suggest that false positive rates vary depending upon the threshold score. Rates of $2 \%, 1 \%$ and $0 \%$ were obtained for the cliniciancomplete version and $7 \%, 2 \%, 1 \%$ for the patient-completed version with respective cut-off scores of 5, 6 and 7. Sensitivity increased as cut off scores were raised as depicted in tables 1 and 2. Bridges et al. (2011) demonstrated a sensitivity of $87 \%$ when using this questionnaire (cut-off $\geq 6$ ) in predicting the outcome of NCS in a cohort of patients with suspected CTS. The cohort of patients in this current study demonstrated a sensitivity of $96 \%$ (clinician-completed version) and $92 \%$ (patient-completed version) using the same cut-off $(\geq 6)$.

The results suggest that if this screening tool was implemented into clinical practice $54 \%$ (using a threshold score of $\geq 6$ ) of those referred with suspected CTS could have avoided onward referral for NCS using the clinician-completed version and $43 \%$ for the patient-completed version.

Whilst the results of this study highlight higher sensitivity rates at all three of the evaluated threshold scores using the clinician-completed questionnaire, we need to consider why one may wish to use the Patient-completed version over the clinician-completed.

An important point to stress is that sensitivity rates using the patient-completed 
versions were very high suggesting a clinical value in its use as a screening tool. The benefit of being able to use this as a patient-completed questionnaire is the improved flexibility, it would allow for its use within primary care. The questionnaire is not designed to be a stand-alone tool in the assessment of CTS it does however add another string to the bow for general practitioners and physiotherapists as an adjunct to their assessment.

If the CTQ was used within primary care, it could be completed prior to referring into specialist services. This would offer potential benefits to both patients and the orthopaedic department. In some circumstance NCS may well be requested at the point of triaging, as hand specialists may well want NCS to be carried out prior to their appointment. Having questionnaire scores on the referral letter will provide the hand specialist further information to indicate which patients should or should not require NCS prior to considering a CTD. The impact of this would potentially reduce the number of referrals for NCS, reducing waiting times, saving money and reducing the potential of the patient being exposed to potentially uncomfortable and unnecessary investigations.

\section{Limitations of the Study}

The participants in this study are already at high risk of presenting with positive NCS as they have all been referred into an orthopaedic clinic having been examined usually in a primary care setting and the suspicion of CTS has been raised. This is reflected in the high positive percentage rate $(75 \%)$ of the results of the NCS. This will have implications when considering the optimum cut-off point and results of this study would not be transferable to a general population.

\section{Conclusions}

Sensitivities obtained within this study for both the patient and clinician completed version of the CTQ strongly support its role as a screening tool for NCS in those patients with suspected CTS. This study increases the flexibility of the tool, providing validity for its use as a patient-completed questionnaire within primary care.

The results obtained from the ROC analysis demonstrate an excellent diagnostic accuracy for both versions furthering the support for the usefulness and practical value of the questionnaire. The potential use of this tool has only been tested on a cohort of patients who have been deemed as high risk of having CTS having already received a preliminary diagnosis of CTS by their GP or another medical practitioner. The generic use of this tool on a low risk population (such as patients presenting with a wide range of functional hand problems in primary care) cannot be judged through the data that have been obtained in this study.

\section{Conflicts of Interest}

The author declares no conflicts of interest regarding the publication of this paper. 


\section{References}

[1] Priganc, V.W. and Henry, S.M. (2003) The Relationship among Five Common Carpal Tunnel Syndrome Tests and the Severity of Carpal Tunnel Syndrome. Journal of Hand Therapy, 16, 225-236. https://doi.org/10.1016/S0894-1130(03)00038-3

[2] Edwards, C. and Frampton, I. (2014) Predicting the Outcome of Nerve Conduction Studies in Patients with Suspected Carpal Tunnel Syndrome: Using an Existing Carpal Tunnel Assessment Tool. Open Journal of Therapy and Rehabilitation, 2, 57-62. https://doi.org/10.4236/ojtr.2014.22010

[3] Levine, D.W., Simmons, B.P., Koris, M.J., Daltroy, L.H., Hohl, G.G., Fossel, A.H. and Katz, J.N. (1993) A Self-Administered Questionnaire for the Assessment of Severity of Symptoms and Functional Status in Carpal Tunnel Syndrome. The Journal of Bone and Joint Surgery, 75, 1585-1592. https://doi.org/10.2106/00004623-199311000-00002

[4] Bridges, M.J., Robertson, D.C. and Chuck, A.J. (2011) Predicting the Result of Nerve Conduction Tests in Carpal Tunnel Syndrome Using a Questionnaire. Hand Surgery, 16, 39-42. https://doi.org/10.1142/S0218810411005047

[5] Kamath, V. and Stothard, J. (2003) A Clinical Questionnaire for the Diagnosis of Carpal Tunnel Syndrome. Journal of Hand Surgery (European Volume), 28, 455-459. https://doi.org/10.1016/S0266-7681(03)00151-7

\section{Appendix. Questionnaire Used within the Study with Scoring Algorithm}

\begin{tabular}{|c|c|c|c|c|}
\hline \multicolumn{5}{|c|}{ Carpal Tunnel Questionnaire } \\
\hline & Question & $\mathrm{Y}$ & $\mathbf{N}$ & N/A \\
\hline 1 & Has pain in your wrist woken you up at night? & 1 & 0 & \\
\hline 2 & Has Tingling or numbness in your hand woken you up at night? & 1 & 0 & \\
\hline 3 & Do you have any trick movements to make the tingling or numbness go from your hands? & 1 & 0 & \\
\hline 4 & Do you have any tingling or numbness in your little finger at any time? & 0 & 3 & \\
\hline 5 & Has tingling or numbness presented when you have been reading a book, steering a car or when knitting? & 1 & 0 & \\
\hline 6 & If applicable has tingling or numbness been severe during pregnancy? & 1 & -1 & 0 \\
\hline 7 & Has it helped the tingling or numbness wearing a splint on your wrist? & 2 & 0 & \\
\hline 8 & Do you have any neck pain? & -1 & 0 & \\
\hline 9 & Has tingling or numbness been more pronounced first thing in the morning? & 1 & 0 & \\
\hline
\end{tabular}

